\title{
Data-based Customer-Retention-as-a-Service: Induktive Entwicklung eines datenbasierten Geschäftsmodells auf Basis einer Fallstudie der Automobilbranche
}

\author{
Henrik Kortum (D) Jonas Rebstadt $\mathbb{D}$ - Laura Sophie Gravemeier $(\mathbb{D})$ \\ Oliver Thomas
}

Eingegangen: 15. Dezember 2020 / Angenommen: 20. März 2021 / Online publiziert: 16. April 2021 (C) Der/die Autor(en) 2021

Zusammenfassung Viele Unternehmen setzen Künstliche Intelligenz zur Verarbeitung großer Datenmengen bereits heute erfolgreich für die Kundenbindung ein. So schaffen große Unternehmen individuelle Kundenerlebnisse basierend auf der Auswertung großer kundenbezogener Datenmengen zur kurz- aber auch langfristigen Kundenbindung, z. B. durch intelligente Empfehlungen von Inhalten auf Videoplattformen. Bei Unternehmen mit traditioneller Wertschöpfung wird dieses Potenzial jedoch noch nicht ausreichend genutzt. Vor diesem Hintergrund wird im Rahmen einer Fallstudie exemplarisch ein datengetriebenes Kundenbindungsszenario in Kooperation mit einer Autowerkstatt umgesetzt. Im konkreten Fall wurde eine zeitlich optimierte Kundenansprache auf Basis von KI-basierten Prognosen der täglichen Fahrleistung von Kunden angestrebt. Grundlage dafür war die Analyse eines Kundendatensatzes einer Autowerkstatt und die anschließende Entwicklung einer Künstlichen Intelligenz. Aufbauend auf der Fallstudie wird ein datenbasiertes Geschäftsmodell konzipiert, dessen Werteangebot vor allem Unternehmen mit traditioneller Wertschöpfung und wenig Wissen im Bereich Künstlicher Intelligenz dazu befähigt, datenbasierte Technologien in der Kundenbindung einzusetzen. Das dem Geschäfts-

\footnotetext{
H. Kortum $(\bowtie) \cdot$ J. Rebstadt $\cdot$ L. S. Gravemeier $\cdot$ O. Thomas

Smart Enterprise Engineering, Deutsches Forschungszentrum für Künstliche Intelligenz GmbH (DFKI), Parkstraße 40, 49080 Osnabrück, Deutschland

E-Mail: henrik.kortum@dfki.de

J. Rebstadt

E-Mail: jonas.rebstadt@dfki.de

L. S. Gravemeier

E-Mail: laura_sophie.gravemeier@dfki.de

O. Thomas

E-Mail: oliver.thomas@dfki.de

O. Thomas

IMWI, Universität Osnabrück, Osnabrück, Deutschland
} 
modell zugrundeliegende Plattformkonzept wird dabei als Open-Innovation-Modell entwickelt und soll neben der Entwicklung eigener Services auch die Interaktion von Datenkonsumenten, Datenlieferanten und anderen Datenbefähigern, mit dem Ziel sich als Datenökosystem für Kundenbindung zu etablieren, unterstützen.

Schlüsselwörter Kundenbindung · Künstliche Intelligenz · Datenbasierte Geschäftsmodelle $\cdot$ Software as a Service $\cdot$ Datenökosystem

\title{
Data-based Customer-Retention-as-a-Service: Inductive Development of a Data-Based Business Model Based on a Case Study of the Automotive Industry
}

\begin{abstract}
Many companies are already successfully using artificial intelligence (AI) to process large volumes of data for the purpose of customer retention. Large companies create individualized customer experiences and analyze massive amounts of data to achieve customer loyalty through intelligent recommendations, for example. However, companies with traditional value creation, as of yet often fail to sufficiently address this topic. Therefore, this contribution tackles the implementation of an exemplary use case for data-driven customer retention in a car repair shop. In particular, the aim was to optimize the timing of customer communication based on forecasts of the customers' daily driving behavior. The basis for this analysis was a data set provided by a car repair shop and the subsequent development of a machine learning model. Based on this case study, a business model is developed that enables companies with traditional value creation and little AI-know-how to use data-driven technologies in customer retention. The underlying platform concept is conceptualized as an open innovation model and supports the interaction of data consumers, data providers and data enablers. In this way, the target is not only to develop own services, but also to establish a data ecosystem for customer loyalty.
\end{abstract}

Keywords Customer Retention · Artificial Intelligence · Data-driven Business Models $\cdot$ Software as a Service $\cdot$ Data Ecosystem

\section{Einleitung}

Big Data und Machine Learning werden von Unternehmen inzwischen in verschiedenen Bereichen gewinnbringend eingesetzt und sind Bestandteil digitaler Geschäftsmodelle (Wiener et al. 2020). Ein wichtiger Bereich ist dabei die Personalisierung von Serviceangeboten sowie die Stärkung der Kundenzufriedenheit und -bindung (Wellers et al. 2017). Für große Tech-Konzerne wie Amazon oder Netflix bilden Big Data und KI-Systeme seit Jahren einen zentralen Faktor ihres wirtschaftlichen Erfolges, indem sie durch die Prognose der „Next Best Action“ und das Aussprechen von Empfehlungen ein individualisiertes Kundenerlebnis für Millionen Nutzer schaffen (Gomez-Uribe und Hunt 2015). Gerade in klassischen Branchen mit weniger digitalen Geschäftsmodellen sind solche Ansätze bisher noch unterrepräsentiert. Neben fehlenden Daten und zu geringer Priorisierung durch das Management (Wiener et al. 
2020), mangelt es hier oftmals am notwendigen Know-How komplexe KI-Services $\mathrm{zu}$ entwickeln und in Unternehmensprozesse zu integrieren (Coleman et al. 2016). Dennoch birgt gerade in solch gering digitalisierten Branchen der Einsatz von KI die Chance einer Wettbewerbsdifferenzierung und das Potenzial, den Kunden durch ein einzigartiges Serviceangebot zu binden. Das Konzept Software-as-a-Service (SaaS) stellt hier einen vielversprechenden Ansatzpunkt dar, um Unternehmen ohne umfassendes KI- und Big-Data-Know-How zu befähigen, das Potenzial dieser Technologien für sich nutzbar zu machen. Die Akzeptanz von SaaS-Geschäftsmodellen ist in den letzten Jahren gestiegen und es ist mit einer zunehmenden Etablierung bis zum Jahr 2025 zu rechnen (Könsgen und Schaarschmidt 2016). Vor diesem Hintergrund entwickeln wir in diesem Beitrag ein plattformbasiertes SaaS-Geschäftsmodell für datenbasierte Kundenbindungsszenarien. Zunächst wird dafür in Kap. 2 die exemplarische Entwicklung eines KI-Services an einem konkreten Use Case demonstriert. Hier beschreiben wir die Umsetzung eines KI-basierten Anwendungsfalles für eine zeitlich gezielte Kundenansprache in einer mittelständischen Autowerkstatt unter Verwendung des Cross-Industry-Standard-Process for Data Mining (CRISP-DM). Darauf aufbauend wird in Kap. 3 ein generisches Geschäftsmodell für eine Kundenbindung-as-a-Service-Plattform anhand eines Business Model Canvas beschrieben. Es folgt eine kritische Diskussion des Geschäftsmodells unter Berücksichtigung einer SWOT-Analyse.

\section{Fallstudie: KI-Service zur Verbesserung der Kundenbindung in einer Kfz-Werkstatt}

\subsection{Hintergrund und Ausgangssituation}

Aktuelle gesellschaftliche und wirtschaftliche Entwicklungen haben weitreichenden Einfluss auf zahlreiche traditionelle Geschäftsmodelle, wovon auch Kfz-Werkstätten betroffen sind. Nicht nur die voranschreitende Digitalisierung, sondern jüngst auch die Auswirkungen der Corona-Krise beeinflussen das Geschäft. Durch die Pandemie arbeiten mehr Menschen im Homeoffice, was eine Reduktion des Autoverkehrs zur Folge hat, weshalb wiederum in Werkstätten weniger Reparaturen und Servicedienstleistungen rund ums Auto verkauft werden. Weiterhin etablieren sich neue Akteure, wie beispielsweise Amazon, auf dem Markt des Ersatzteilgeschäftes. Dementsprechend sind etablierte Werkstätten einem steigenden Wettbewerbsdruck ausgesetzt. Folglich steht eine Anpassung und Modernisierung der bestehenden Geschäftsmodelle aus (Alfs und Deckers 2020). Daher wird in dieser Fallstudie in Kooperation mit einer Autowerkstatt untersucht, inwiefern KI-basierte Verfahren zur Auswertung von Kundendaten traditionelle Werkstätten in Kundenbindungsszenarien unterstützen können. Ziel ist es datenbasierte Empfehlungen ausgesprochen, um Kunden zum richtigen Zeitpunkt zu erreichen.

In der betrachteten Werkstatt gehört das Anbieten von wiederkehrenden Servicedienstleistungen wie Öl-Wechseln zum Geschäftsmodell. Abhängig vom Fahrzeugmodell sind definierte Serviceintervalle festgelegt, die sich alle an der Fahrleistung der Kunden orientieren. Laut Aussage der befragten Domänenexperten stellt die 
Fahrleistung die zentrale Steuerungsgröße für das Anbieten von Servicedienstleistungen dar. Diese können aktuell nur dann angeboten werden, wenn Kunden selbst auf die Werkstatt zugehen oder für eine Reparatur zufälligerweise zum passenden Serviceintervall vor Ort sind. Servicedienstleistungen im Rahmen des Serviceplans stellen darüber hinaus eine sehr gute Möglichkeit dar, ein Cross-Selling für andere Dienstleistungen, wie beispielsweise das Wechseln von Bremsscheiben durchzuführen, wenn dies notwendig ist. Vor diesem Hintergrund ist die gezielte und aktive Ansprache der Kunden sowie das Angebot entsprechender Dienstleistungen beim Anfall eines Serviceintervalls sinnvoll. Derartige Kundenansprachen bleiben derzeit jedoch bei unabhängigen Werkstätten aus, da die Information über das kundenindividuelle Fahrverhalten fehlen. Daher ist das Ziel dieser Fallstudie die datenbasierte Prognose des Fahrverhaltens der Kunden. Auf Basis der prognostizierten Fahrleistung kann die Werkstatt den nächsten notwendigen Servicetermin ermitteln, um ihre Servicedienstleistungen dann anzubieten, wenn diese auch benötigt werden.

\subsection{Vorgehen zur Entwicklung von KI-Systemen}

Ein etabliertes Vorgehen für die Umsetzung von datenbasierten Anwendungsfällen ist der CRISP-DM (Shearer 2000). Ursprünglich dient der CRISP-DM der Umsetzung von Data-Mining-Projekten, wird jedoch erfolgreich in KI-Projekten instrumentalisiert (Overgoor et al. 2019). Der CRISP-DM besteht aus insgesamt sechs Schritten: Business Understanding, Data Understanding, Data Preparation, Modeling, Evaluation und Deployment (Shearer 2000). Im ersten Schritt, Business Understanding, gilt es die Ziele aus Business- sowie Datensicht zu konkretisieren. So werden Rahmenbedingungen, wie z. B. Anforderungen und Erfolgskriterien an das System definiert. Ziele des zweiten Schrittes, Data Understanding, sind die initiale Beschaffung bzw. das Sammeln von Daten, die Beschreibung und erste Analyse dieser sowie die Dokumentation der Datenqualität. Im dritten Schritt, Data Preparation, werden die Daten für das Modeling vorbereitet. Beim Modeling besteht die Aufgabe unter anderem in der Auswahl der richtigen Modellierungsmethode, der Auswahl von Modellen sowie der Einstellung von Parametern. Die Schritte der Data Preparation und des Modeling werden iterativ durchgeführt, sodass eine immer weitere Verfeinerung der Ergebnisse möglich ist. Nach Abschluss des Modeling werden in der Phase Evaluation die Ergebnisse in Hinblick auf die Geschäfts- und Datenziele bewertet. Werden alle Anforderungen erfüllt, kann das Deployment angestoßen werden. Die Produktivsetzung der Anwendung für den praktischen Einsatz wird in diesem Schritt geplant und durchgeführt (Shearer 2000; Overgoor et al. 2019).

\subsection{Business Understanding}

Im Rahmen eines Fokusgruppengespräches, durchgeführt mit Domänenexperten aus einer Kfz-Werkstatt, Data Scientisten und Softwareentwicklern, wurde das Anwendungsszenario der gezielten Kundenansprache konkretisiert und Anforderungen an das dafür benötigte KI-System identifiziert.

Das Businessziel des vorliegenden Anwendungsfalles ist eine zeitlich verbesserte Kundenansprache, welche über eine Prognose der Fahrleistung von Kunden 
erfolgen soll. Hintergrund ist, dass bei jedem Fahrzeug vom Hersteller festgelegte Inspektionen nach bestimmten Intervallen anfallen. Bei jedem Fahrzeug ist damit vorgeschrieben, zu welchem Kilometerstand welche Serviceleistungen durchgeführt werden müssen. Das Fahrzeug gibt dem Fahrzeugführer über den Bordcomputer Informationen darüber, wann der nächste Service fällig ist. Diese Information liegt den freien Autowerkstätten nicht explizit vor. Zwar können sie Informationen dazu beschaffen, bei welchem Modell zu welchen Kilometerständen welche Serviceleistungen anfallen, allerdings fehlen die Informationen zur Fahrleistung der Kunden. Hier besteht ein Wettbewerbsnachteil gegenüber Vertragswerkstätten, da diese die Information der Kilometerstände von den Fahrzeugen übermittelt bekommen. Damit sind Vertragswerkstätten in der Lage, proaktiv auf den Kunden zuzugehen und ein Servicetermin anzubieten. Um freie Werkstätten ebenfalls zur zeitlich gezielteren Kundenansprache zu befähigen, kann eine Prognose der aktuellen Laufleistung des Fahrzeuges instrumentalisiert werden. Das aus dem Business-Ziel abgeleitete Analyseziel ist es daher, auf Basis historischer Kundendaten die durchschnittliche Laufleistung $(\mathrm{km} / \mathrm{Tag})$ eines Kundenfahrzeuges zu prognostizieren. Die Zielvariable $(\mathrm{km} / \mathrm{Tag})$ liegt in den Daten nur implizit vor und muss in späteren Schritten aus dem Datensatz berechnet werden. Die Prognose dient in Kombination mit den vorgeschriebenen Serviceintervallen als Indikator für einen verbesserten Zeitpunkt zur Kundenansprache. Als fachlicher Benchmark wurde durch die Domänenexperten die durchschnittliche Fahrleistung i. H.v. $43 \mathrm{~km} / \mathrm{Tag}$ genannt, die linear über das Jahr fortgeschrieben wird. Aus fachlicher Sicht ist eine Überschätzung der Fahrleistung weniger kritisch als eine Unterschätzung. Erstgenannter Fall könne als Kundenbindungsmaßnahme und für eine Terminvereinbarung in der Zukunft genutzt werden, während eine Unterschätzung mit einer entgangenen Vertriebschance gleichzusetzen sei, so die Experten. Weiterhin wurden Anforderungen für eine wirtschaftliche Verwendung der KI-Applikation formuliert. Eine wesentliche Anforderung ist die Automatisierbarkeit und Interoperabilität, damit die KI-Applikation in die bestehenden Prozesse und Systeme eingebunden werden kann. Eine weitere Anforderung gilt dem Datenschutz. Zur Validierung und zur gezielten Nutzung der prognostizierten Kilometerstände wurde eine grundsätzliche Erklärbarkeit der Prognosen als Anforderung aufgenommen.

\subsection{Data Understanding \& Data Preparation}

Datengrundlage für die Umsetzung ist ein anonymisierter Kundendatensatz aus dem ERP-System einer mittelständischen Autowerkstatt. Die Daten wurden im Zeitraum von 2005 bis 2019 erhoben und liegen in tabellarischer Form vor (Datenpunkte von 47.415 Fahrzeugen mit jeweils bis zu 47 Attributen). In der Data UnderstandingPhase wurden die vorliegenden Attribute analysiert. Der Fokus hierbei lag auf dem Verständnis der unterliegenden Verteilungen, der Identifikation von Attributen mit Ausreißern und dem Offenlegen von Korrelationen sowohl zwischen verschiedenen Input-Attributen als auch von Input-Attributen zum durch den Algorithmus zu lernendem Zielwert ( $\mathrm{km} / \mathrm{Tag}$ ). Basierend auf diesen Analysen wurde ein Data Preparation initialisiert, in welchem die Schritte Data Cleaning, Data Selection, Data Format, Data Construction und Data Integration nach Pérez et al. (2015) durch- 
laufen wurden. Im Data Cleaning wurden auf Basis des Kennzeichens und einer eindeutigen Fahrzeug-ID doppelte Einträge gelöscht sowie identifizierte und durch Handwerker validierte Ausreißer entfernt. In der Phase Data Selection wurden doppelt enthaltene sowie nicht gefüllte Attribute entfernt. Die als relevant identifizierten Attribute wurden im nächsten Schritt, dem Data Format, von dem initialen und eher allgemeinen Typ (Object) in die passenden Daten-Formate (String, Integer, Float, Boolean) transformiert. Im Rahmen der Data Construction wurde aus den vorliegenden Rohdaten die Zielvariable tägliche Fahrleistung generiert sowie weitere erklärende Attribute gebildet. Das relevanteste Beispiel in dem Anwendungsfall ist neben den Ortsinformationen des Nummernschildes die Fahrzeug-Identifizierungsnummer. Die Inhalte der Identifikationsnummern wurden, abgesehen von kleinen Änderungen 2011, bereits 1975 stellengenau vorgegeben. ${ }^{1}$ Hierdurch konnten für das Modell an den Positionen 1-3 und 12-14 der Welt-Herstellercode, an den Positionen 4-9 die Baureihe des Herstellers, an Stelle 10 der Modelljahrescode und an Stelle 11 das Herstellerwerk extrahiert werden. ${ }^{2}$ Die hierbei entwickelten Attribute wurden im Folgenden in drei Kategorien unterteilt: (1) Attribute, die spezifisch nur für diesen konkreten Anwendungsfall vorliegen, (2) allgemeine Attribute, die 1t. Fokusgruppenbefragung in den meisten Werkstätten vorliegen müssten, (3) allgemeine Attribute, die in jeder Werkstatt erwartet werden.

Im finalen Schritt, der Data Integration, wurden die erstellten Attribute in einer gemeinsamen Datenstruktur zusammengeführt und in ein einheitliches Format gebracht. Bei der Data Preperation haben sich vor allem zwei anwendungsfallspezifische Herausforderungen herauskristallisiert. Zum einen existieren stark miteinander korrelierende Parameter wie PS und KW sowie die Herstellerangabe und der WeltHerstellercode (WMI-Kennung), welche eine unabhängige Betrachtung durch die eingesetzten Algorithmen sowie die spätere Erklärbarkeit des vorhergesagten Ergebnisses auf Basis der relevanten Attribute erschwert. Die betroffenen Variablen wurden mithilfe einer Korrelationsanalyse identifiziert und eine der gefundenen Variablen wurde aus dem Datensatz entfernt. Zum anderen liegen in dem Datensatz deutliche Unterschiede bezüglich der Anzahl an angegebenen Kilometerständen vor. Aufgrund der generell nur spärlich gefüllten Variablen konnte im Zuge der Analyse nicht auf für Zeitreihen typische Verfahren wie Sliding-Windows zurückgegriffen werden. Vielmehr wurden die existierenden Angaben zu Durchschnittswerten aggregiert, womit zudem auch eine Zusammenführung der Kilometerdaten mit den übrigen Variablen erleichtert wurde.

\subsection{Modeling}

Das Modell zur Prognose der Fahrleistung wurde als Regressionsproblem formuliert, wobei als Zielvariable die durchschnittliche tägliche Fahrleistung in $\mathrm{km} / \mathrm{Tag}$ diente. Für dessen Lösung fand die Python-Implementierung des CatBoost Algorithmus (Xia et al. 2017) Anwendung. CatBoost nutzt Gradient Boosting auf Entscheidungsbäumen und liefert State-of-the-Art-Ergebnisse im Machine-Learning-

1 Vgl. https://eur-lex.europa.eu/legal-content/DE/TXT/?uri=CELEX:31976L0114.
2 Vgl. https://eur-lex europa.eu/legal-content/DE/TXT/? uri=CELEX:32011R0019. 


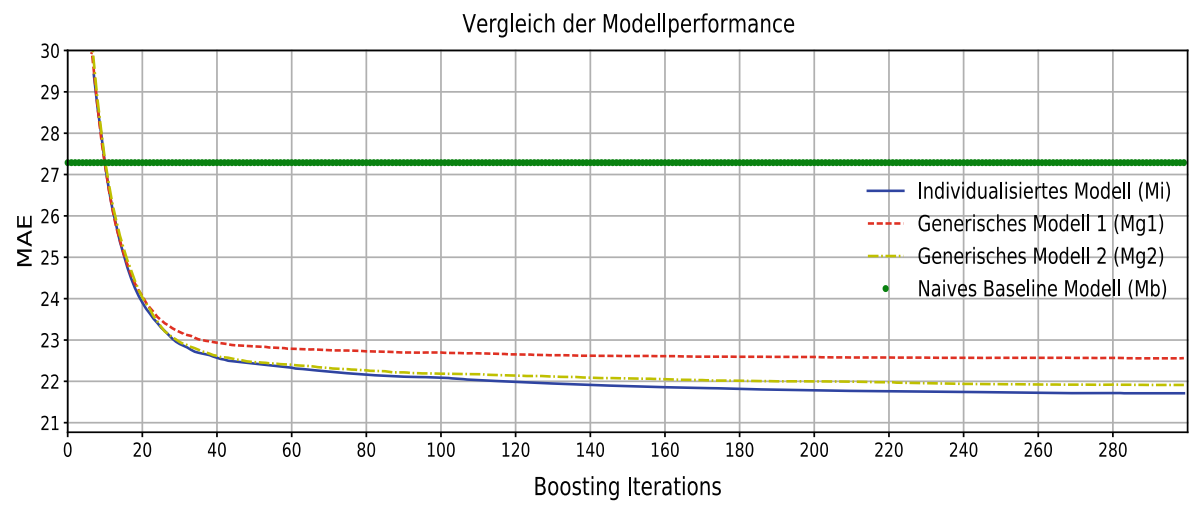

Abb. 1 Vergleich des mittleren absoluten Testfehlers des individualisierten Modells, der beiden generischen Modelle und eines einfachen Basismodells

Bereich. Mit diesem Verfahren wurden drei Modelle trainiert: Ein individualisiertes Modell $\mathrm{M}_{\mathrm{i}}$, welches werkstattspezifische Variablen nutzt und zwei generische Modelle $\mathrm{M}_{\mathrm{g} 1}$ und $\mathrm{M}_{\mathrm{g} 2}$, die auf allgemeinen Werkstattdaten trainiert wurden. Abb. 1 zeigt die Modellperformance auf Basis des mittleren absoluten Testfehlers (MATeE) der drei Modelle in Abhängigkeit der für das Training verwendeten Iterationen. Das Modell $\mathbf{M}_{\mathrm{i}}$ erreichte einen MATeE von 21,55, das Modell $\mathbf{M}_{\mathrm{g} 1}$ einen MATeE von 21,77 und das Modell $\mathrm{M}_{\mathrm{g} 2}$ erreichte einen MATeE von 22,55. Ein naives Baseline-Schätzmodell $\mathrm{M}_{\mathrm{b}}$, welches lediglich die durchschnittliche tägliche Fahrleistung i. H.v. 43,06 km/Tag als Schätzer heranzieht, verfügt über einen mittleren Fehler von 27,29 .

\subsection{Evaluation}

Zur statistischen Absicherung dieser Ergebnisse wurde ein Wilcoxon-VorzeichenRang-Test (Wilcoxon et al. 1970) für gepaarte Stichproben durchgeführt. Dabei wurde der Fehler von je zwei Modellen für die Samplesplits des Testdatensatzes miteinander verglichen. Es ergibt sich für sämtliche Modelle ein zu allen gängigen Signifikanzniveaus geringerer Fehler, als sich bei Verwendung eines naiven Schätzmodells auf Basis des Mittelwerts der Fahrleistung ergeben würde. Selbst das einfache generische Modell $\mathrm{M}_{\mathrm{g} 2}$, das als Parameter nur die Fahrleistung seit Erstzulassung und eine Fahrgestellnummer nutzt, führt zu einer Reduktion des mittleren Fehlers von über $20 \%$. Die KI-basierten Prognosemodelle zeigen damit eine signifikant bessere Vorhersage der durchschnittlichen täglichen Fahrleistung als eine naive Herangehensweise. Zusätzlich zu den berechneten Kilometerleistungen wurde mithilfe der Feature Importance eine Erklärbarkeit der Prognosen auf Instanzebene hergestellt um 1) eine fachliche Verifizierung des Modells zu erreichen und 2) eine bessere Einschätzung der Ergebnisse und somit effektivere Nutzung durch die Autowerkstätten zu ermöglichen. Die Relevanzberechnung der einzelnen Features erfolgt hierbei auf Basis von SHAP-Values (Lundberg und Lee 2017), welche spieltheoretisch fundiert den Beitrag der einzelnen Features auf die Gesamt-Vorhersage 


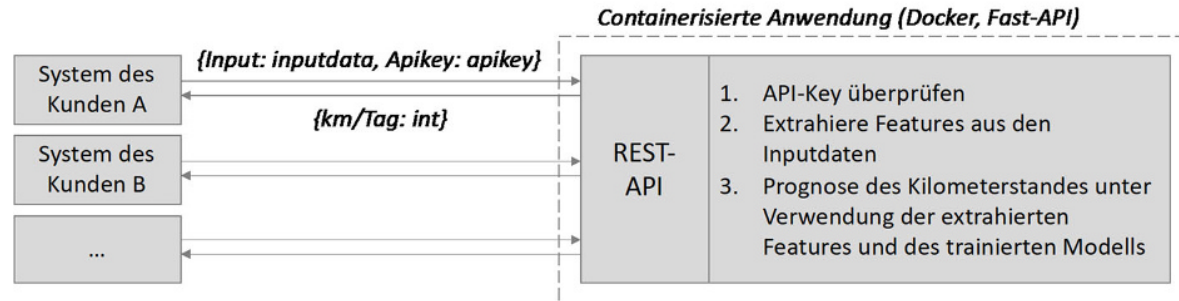

Abb. 2 Schematische Darstellung der Schnittstellenarchitektur des Prognoseservice

herleiten. Die Modellergebnisse wurden den Domänenexperten vorgestellt und hinsichtlich eines Praxiseinsatzes evaluiert. Alle drei Modelle stellen aus unternehmerischer Sicht der Experten (unter Vernachlässigung der Kosten für die Entwicklung und Implementierung) einen Mehrwert dar, da sie im vorliegenden Anwendungsfall besser abschneiden als das naive Baseline-Modell. Für den Praxiseinsatz sind zudem Prognosen für Kunden irrelevant, die eine jährliche Fahrleistung von $15.000 \mathrm{~km}$ (ca. $41 \mathrm{~km} / \mathrm{Tag}$ ) unterschreiten, da in diesem Fall standardmäßig eine Kontaktaufnahme für eine Jahresuntersuchung erfolgen würde. Im Falle der durchschnittlichen jährlichen Fahrleistung i.H.v. $15.695 \mathrm{~km}$ bedeutet das, dass durch das Modell für ca. $50 \%$ der Kunden ein neuer potenzieller Vertriebsanlass geschaffen werden kann.

\subsection{Deployment}

Entsprechend der im Fokusgruppengespräch definierten Anforderungen wird das KI-Modell über eine REST-Schnittstelle bereitgestellt, deren Architektur in Abb. 2 dargestellt ist.

Durch die Implementierung als API wird die Interoperabilität mit bereits vorhandenen Systemen gewährleistet. Um die Anforderung an den Datenschutz zu erfüllen, ist die REST-API über einen API-Key gesichert. Damit haben nur autorisierte Nutzer Zugang zur Schnittstelle. Daneben werden vom KI-Service keine Daten gespeichert und nur temporär während der Berechnung der Prognose im Arbeitsspeicher gehalten. Technisch wurde das Deployment mithilfe von Docker und des Python-Moduls Fast-API realisiert. Aufgrund der gewählten Architektur ist eine skalierbare Bereitstellung problemlos möglich. Die Anwendung kann beispielsweise in wenigen Schritten in der Cloud bereitgestellt werden. Damit liegt eine Lösung vor, die von anderen Werkstätten genutzt werden kann. Mit dieser Rest-API wird der KI-Service für die Fachsysteme der Werkstätten zugänglich gemacht, um Vertriebs- und Serviceprozesse zu unterstützen. Im Rahmen der Fokusgruppe wurde eine daran angeschlossene Integration in CRM- oder Leadmanagement-Systeme diskutiert. Hier erhalten die Servicemitarbeiter der Werkstatt Leads, wenn bevorstehende Serviceintervalle bei bestimmten Kunden prognostiziert werden. Zudem wurde die automatisierte, direkte Ansprache der Kunden per Mail oder Textnachricht an den Fahrzeughalter zur Terminvereinbarung als Anwendungsszenario identifiziert. 


\section{Skalierbares Geschäftsmodell: KI-Service-Plattform zur Kundenbindung}

Diese prototypische Implementierung eines KI-Services zur Steigerung der Kundenbindung dient dabei nicht nur als Lösungsvorschlag des vorliegenden Anwendungsfalls, sondern auch als Basis für die induktive Ableitung eines übergeordneten OpenInnovation-Ansatzes. Hierdurch können gerade kleinere Unternehmen zu Innovationen befähigt werden, welche aus Kosten und Risikoaspekten ansonsten nicht hätten abgebildet werden können (Gassmann und Enkel 2006). Vor allem in den Bereichen der datenbasierten Wertschöpfung fehlt es kleinen und mittleren Unternehmen häufig an Know-How und den notwendigen Ressourcen, die Potenziale datenbasierter Wertschöpfung eigenständig in ihr Geschäftsmodell zu integrieren (Rimbeck et al. 2020). Der Fokus liegt hierbei nicht nur auf der Zusammenarbeit konkurrierender und komplementärer Unternehmen, sondern viel mehr auf einer Integration aller relevanten Akteure wie Lieferanten, Forschungsinstitute und Kunden (Gassmann und Enkel 2006). Die Entwicklung und das Angebot von datenbasierten Services für Dritte entspricht dem Geschäftsmodell eines Datenbefähigers (Wiener et al. 2020). Ein Datenbefähiger ermöglicht es Datenkonsumenten - z. B. Autowerkstätten - die Vorteile datenbasierter Services zu nutzen, ohne dass diese selbst größeres KnowHow und Ressourcen in diesem Bereich aufbauen müssten. In den vorangegangenen Kapiteln konnten wir zeigen, dass die Nutzung eines datenbasierten Kundenbindungsservice auch für kleine Kfz-Werkstätten großes Vertriebspotenzial birgt. An dieser Stelle setzt das durch uns entwickelte Geschäftsmodell an, welches KIund Software-Know-How zu Kundenbindungszwecken gebündelt als SaaS-Lösung auf einer Plattform zur Verfügung stellt. Hier werden allgemeine Lösungen für optimierte Maßnahmen zur Kundenbindung entwickelt, die innerhalb einer Branche übertragbar sind. Gleichzeitig können auch individuell an spezifische Kundenunternehmen angepasste Lösungen entwickelt werden. Neben der Bereitstellung technischer Services wird auch das Potenzial der Plattform als Datenökosystem diskutiert. Datenökosysteme fördern das Zusammenspiel verschiedener Akteure, wie z. B. Organisationen und Einzelpersonen, die Daten in Netzwerken bereitstellen, transformieren, als Ressourcen nutzen und Dienstleistungen in diesem Umfeld anbieten (Oliveira und Lóscio 2018). Somit verbinden Datenökosysteme die Geschäftsmodelle von Datennutzern, Datenlieferanten und Datenbefähigern (Wiener et al. 2020) miteinander. Im Rahmen der Fokusgruppe wurden daher nicht nur Rahmenbedingungen und Anforderungen des oben beschriebenen Anwendungsfalls definiert, sondern auch die Strukturierung und Konkretisierung eines skalierbaren, plattformbasierten Geschäftsmodells anhand des Business Model Canvas Frameworks (Osterwalder und Pigneur 2010) vorgenommen (siehe Abb. 3).

Bei den Werteangeboten des Geschäftsmodells ist zwischen direktem Kundennutzen (B2B) und dem indirekten Nutzen für die Endkunden (B2B2C) zu differenzieren. Der direkte Geschäftskunde kann als Datenkonsument mithilfe der über die Plattform angebotenen Dienstleistungen eine höhere Endkundenbindung erreichen. So können mithilfe von KI-Services z. B. gezieltere Zeitpunkte für eine Kundenansprache oder Produkte für ein Cross Selling vorgeschlagen werden. Ziel der Datenkonsumenten ist es eine höhere Endkundenbindung aufzubauen, die Loyali- 


\begin{tabular}{|c|c|c|c|c|}
\hline $\begin{array}{ll}\text { Schlüsselpartner } \\
\text { - } & \text { Verschiedene } \\
& \text { Branchennetzwerke } \\
\text { - } & \text { Cloud-Plattform- } \\
& \text { Betreiber } \\
\text { - } & \text { Datenlieferanten } \\
\text { - Datenkonsumenten } & \text { Weitere } \\
\text { - } & \text { Datenbefähiger }\end{array}$ & 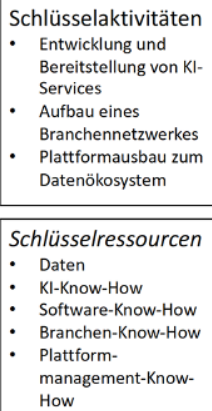 & $\begin{array}{l}\text { Werteangebote } \\
\text { Service für die Plattformnutzer } \\
\text { (Unternehmen) B2B: } \\
\text { - Datenkonsumenten: } \\
\text { Zugang zu KI-Services zur } \\
\text { Kundenbindung } \\
\text { - Datenlieferanten: } \\
\text { Monetarisierung ihrer } \\
\text { Daten über die Plattform } \\
\text { - Andere Datenbefähiger: } \\
\text { Markt für ihrer Services } \\
\text { Service für die Endkunden } \\
\text { B2B2C: } \\
\text { - Bedarfsgerechtere } \\
\text { Kundenansprache } \\
\text { - Verbesserter } \\
\text { Serviceleistungen } \\
\text { - Komfort }\end{array}$ & 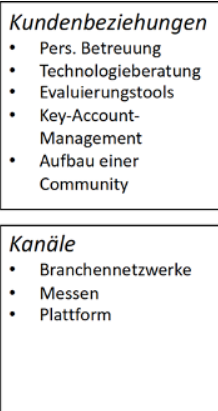 & $\begin{array}{ll}\text { Kundensegmente } \\
\text { - Datenkonsumenten } \\
\text { (Erwerben } \\
\text { Datenservices über } \\
\text { die Plattform) } \\
\text { - Datenlieferanten } \\
\text { - Datenbefähiger }\end{array}$ \\
\hline \multicolumn{2}{|c|}{$\begin{array}{l}\text { Kostenstruktur } \\
\text { - } \quad \text { Kosten für Personal } \\
\text { - } \quad \text { Kosten für Cloudnutzung und Plattformmanagement } \\
\text { - }\end{array}$} & \multicolumn{3}{|c|}{$\begin{array}{l}\text { Einnahmequellen } \\
\text { : } \text { Abonnements } \\
\text { - Pay-per-use } \\
\text { Beteiligung an Transaktionen der Plattformnutzer }\end{array}$} \\
\hline
\end{tabular}

Abb. 3 Darstellung des KI-Service-Plattform-Geschäftsmodells als Business Model Canvas

tät der Endkunden zu steigern und die Kundenabwanderung zu vermeiden. Auch Endkunden profitieren bei diesem Wertschöpfungsmodell vom Werteangebot durch eine Steigerung des Komforts: sie erhalten individualisierte Betreuung, attraktivere Angebote sowie Dienstleistungsvorschläge. Darüber hinaus bietet die Plattform Datenlieferanten die Möglichkeit, ihre Daten und zugehörige Services gebündelt einer breiten Menge an Kunden anzubieten. Andere Serviceanbieter (Datenbefähiger) erhalten durch die Plattform eine Möglichkeit ihre Services Datennutzern zu offerieren. Um dieses Werteangebot herzustellen, werden wesentliche Partner und Ressourcen benötigt. Daneben sind Schlüsselaktivitäten durchzuführen. Als Schlüsselpartner wurden verschiedene Branchennetzwerke, Cloud-Plattform-Betreiber und Datenlieferanten sowie -nutzer aber auch weitere Datenbefähiger bzw. Serviceanbieter identifiziert. Über einen stetigen Austausch mit Branchennetzwerken und Spezialisten aus den Kernbranchen können individuelle Bedürfnisse abgefragt sowie branchenspezifisches Know-How aufgebaut werden. Cloud-Plattform-Betreiber sind ein weiterer Schlüsselpartner, da durch Cloud-Plattformen eine Möglichkeit zur Verfügung steht, die eigenen digitalen Services oder auch die gesamte KI-ServicePlattform zu hosten und die verschiedenen Datenquellen bereitzustellen. Da das Geschäftsmodell einen Open-Innovation-Ansatz verfolgt, lassen sich ex ante nicht sämtliche potenziell relevanten Partner abschließend identifizieren. Im Laufe der Zeit werden sich die Plattform und deren Nutzergruppen weiterentwickeln. Damit sich die Plattform zu einem attraktiven Datenökosystem entwickeln kann, sollten alle Interessierten möglichst einfach Zugang erhalten können. Nur wenn über das Ökosystem der Plattform genügend Daten zur Verfügung stehen, können die entsprechenden KI-Services entwickelt werden. Deswegen stellen Daten die wichtigste Schlüsselressource der Plattform dar. Es sind verschiedene Szenarien der Datenakquise denkbar. Einerseits könnte für konkrete Services ein „bring your own data“ Ansatz verfolgt werden, bei dem für einen Konsumenten einen Service gezielt auf Basis seiner eigenen Daten entwickelt wird. Andererseits birgt die Plattform aber gerade das Potenzial Daten verschiedener Datenlieferanten zu bündeln, um auf dieser Basis bessere Modelle zu entwickeln und so weitere Anreize für die Plattformnut- 
zung zu schaffen. Um Datenlieferanten dazu zu bringen, Daten auf der Plattform bereitzustellen, könnten sie z. B. monetär an den Erlösen der Services, die ihre Daten nutzen, beteiligt werden. Weitere Schlüsselressourcen sind KI-, Software- und Branchen-Know-How sowie Fähigkeiten im Bereich des Plattformmanagements. Damit die Plattform initial Nutzer in Form von Datenlieferanten und Datennutzern gewinnen kann, muss sie über ein Basisangebot an Services verfügen. Nach der Etablierung der Plattform und der Ansiedlung von weiteren Service-Anbietern (Datenbefähigern) rückt die Notwendigkeit der Entwicklung eigener KI-Services in den Hintergrund und das Plattformmanagement gewinnt an Bedeutung. Die Schlüsselaktivitäten zielen darauf ab, a) die KI-Services zu entwickeln und bereitzustellen, b) den Aufbau von Branchen-Know-How voranzutreiben, um die Perspektive des Kundennutzens nicht zu vernachlässigen und in den Vordergrund zu rücken und c) den Aufbau der Plattform in Richtung eines Datenökosystems voranzutreiben. Damit verbunden ist die Kostenstruktur des Geschäftsmodells, wobei die wesentlichen Kosten für qualifiziertes Personal für die Service Entwicklung und das Plattformmanagement anfallen. Weiterhin entstehen nutzungsabhängige Kosten für die Cloud-Nutzung und für die Akquisition von Plattformnutzern und Daten. Dagegen stehen die Einnahmen, welche sowohl über Pay-per-Use-Modelle als auch Abonnements für eigene Services und die Plattformnutzung erzielt werden können. Zudem erfolgt eine Beteiligung der Plattformbetreiber an den Erlösen, die durch Services Dritter erbracht und über die Plattform angeboten werden. Mit fortschreitendem Wachstum der Plattform gewinnt vor allem die Beteiligung an Transaktionen Dritter an monetärer Bedeutung. Potenzielle Kunden stellen in diesem Geschäftsmodell alle Unternehmen dar, die ein wiederkehrendes Angebot von Produkten oder Services anbieten und zu deren Unterstützung Datenservices über die Plattform erwerben. Vor allem Unternehmen, bei denen traditionelle Wertschöpfung im Fokus steht, sind Zielgruppe des Geschäftsmodells, da sie nicht über das Know-How verfügen, diese selbst zu entwickeln. Daneben sind aber auch die Schlüsselpartner der Kategorien Datenlieferanten und Datenbefähiger als Kunden des Geschäftsmodells zu verstehen. Die Kunden sollen über verschiedene Kanäle erreicht werden: über die Plattform selbst, aber auch über Branchennetzwerke und Messen. Um eine langfristige und gute Basis für die Kundenbeziehung aufzubauen, steht der persönliche Kontakt zu den Kunden vorerst im Vordergrund. Die Kunden werden bei der Technologie- und Serviceauswahl unterstützt und es werden Evaluierungstools zur Verfügung gestellt, damit der Nutzen der Plattform messbar wird. Über ein Keyaccount Management soll der Aufbau der Plattform zum Datenökosystem gezielt vorangetrieben werden. Auch sollte die Plattform selbst Raum für Austausch und Kommunikation zwischen Akteuren des Ökosystems bieten, damit sich eine Plattformcommunity entwickelt.

\section{Diskussion}

Um das Geschäftsmodell im Detail zu analysieren, wurde eine SWOT-Analyse durchgeführt. Die Stärken des Geschäftsmodells bestehen im hohen Skalierbarkeitspotenzial in Verbindung mit den geringen Investitions- und Betriebskosten. Ermöglicht wird dies durch die Nutzung von Cloudinfrastruktur und der Umsetzung von ge- 
neralisierbaren Anwendungsfällen. Eine weitere Stärke des Geschäftsmodells ergibt sich aus der hohen thematischen Relevanz von Kundenbindung für Unternehmen. Besonders deutlich wird dies vor dem in der Fallstudie beschriebenen Hintergrund, dass Unternehmen ihre herkömmlichen Geschäftsmodelle überdenken müssen, um sich vom Wettbewerb zu differenzieren. Mithilfe von Kundenbindung-as-a-Service können Unternehmen einer Art Differenzierung gerecht werden und sich gleichzeitig auf ihre Kernkompetenzen konzentrieren. Eine potenzielle Schwäche des Geschäftsmodells ist das Fehlen von eigenem Datenbesitz und damit ein erschwerter Zugang zu Daten als Ressource. Das Datenökosystem der Plattform verfügt im Startzeitpunkt nicht über hinreichende Datenmengen, um eine breite Masse an Services umzusetzen. Hinzu kommt fehlendes Branchen-Know-How der Plattformbetreiber, was die Identifikation relevanter Anwendungsfälle erschwert. So sind die Wettbewerbsnachteile unabhängiger Werkstätten im Vergleich zu Vertragswerkstätten, die zentrale Faktoren im beschriebenen Anwendungsfall darstellen, Software- und KIEntwicklern nicht zwingend bekannt. Chancen für das Geschäftsmodell ergeben sich vor allem aus der potenziellen Weiterentwicklung der Plattform in ein vollwertiges Datenökosystem. Aufgrund der zunehmenden Bedeutung von Daten als Rohstoff zeichnet sich die Tendenz für die Etablierung von Datenmarkplätzen bereits heute ab (Lange et al. 2018). Auf diese Weise wird der Zugang zu verschiedensten Datenquellen ermöglicht. Im Optimalfall tritt so ein selbstverstärkender Effekt ein: Je mehr Daten durch Datenlieferanten bereitgestellt werden, um so besser sind die durch Datenbefähiger entwickelten KI-Services, die von um so mehr Datenkonsumenten genutzt werden, was wiederum zu größeren Umsatzpotenzialen der Plattform-Services führt und damit die Attraktivität der Datenbereitstellung für Datenlieferanten erhöht. In Zusammenhang mit dem Zugang zu mehreren Datenquellen steht auch die Skalierbarkeit des Geschäftsmodelles auf den gesamten Prozess der Customer Journey. Im aktuellen Konzept ist die Kundenbindungsplattform der Phase Retention zuzuordnen. Eine Ausweitung des Konzeptes auf die Phasen Awareness, Consideration, Purchase sowie Advocacy ist denkbar. Der weitgehend offene Plattformansatz fördert das Aufkommen von Innovationen und damit die Weiterentwicklung des Geschäftsmodells. Ein Risiko besteht darin, dass nicht genügend generalisierbare Anwendungsfälle analog zur Prognose der täglichen Fahrleistung identifiziert werden. Dann besteht die Gefahr, dass keine skalierbare Plattform entsteht. Ein weiteres Risiko stellt eine möglicherweise fehlende Akzeptanz der angebotenen Dienstleistung bei Unternehmen dar. Zielgruppe sind Unternehmen, die zwar nicht per se einen hohen digitalen Reifegrad aufweisen, aber den Bedarf für KI-basierte Dienstleistungen erkennen. Dennoch stellt eine zu geringe Digitalisierung bei Kundenunternehmen ein weiteres Risiko des Geschäftsmodells dar, da die Verwendung der hier konzipierten Kundenbindungs-Plattform einen gewissen Digitalisierungsgrad voraussetzt. Weiterhin besteht das Risiko, dass potenzielle Datenlieferanten, die zur Entwicklung von KI-Services benötigten Daten trotz monetärer Anreize nicht teilen wollen. Allgemein besteht das Risiko, dass die Plattform nicht die kritische Masse an Teilnehmern erreicht, die notwendig ist, um sich als Datenökosystem zu etablieren.

Auf Basis der identifizierten Ergebnisse lassen sich verschiedene Strategien ableiten. (1) Ausbauen: Als SO-Strategie (Stärken-Chancen-Kombinationen) kann die Erweiterung des Dienstleistungsportfolios auf den gesamten Prozess der Customer 
Journey dienen. Unter der Voraussetzung von geringen Investitions- und Betriebskosten stellt diese Ausdehnung des Geschäftsmodells eine risikoarme, aber erfolgversprechende Option dar. Zudem sollte der Fokus auf dem breiten Ausbau der Plattform als Datenökosystem und der Gewinnung von Plattformnutzern gelegt werden, um möglichst schnell eine kritische Masse zu erreichen. (2) Absichern: Im Sinne einer SR-Strategie (Stärken-Risiken-Kombination) ist aber auch eine Spezialisierung möglich. Die Gefahr fehlender generalisierbarer Anwendungsfälle wird durch die geringen Kosten abgefangen. In dieser Strategie gilt es, den globalen Anspruch des Geschäftsmodells einzuschränken und den Fokus auf wenig digitalisierte Branchen zu legen. Eine weitere Spezialisierung kann durch das Anbieten von individualisierten KI-Services erfolgen, wie es beispielsweise im Anwendungsfall der gezielten Kundenansprache der Kfz-Werkstatt der Fall ist. Im Falle einer Fokussierung sollte auf eine hohe Qualität der angebotenen Services in Kombination mit einem Key Account Management für die gezielte Gewinnung von Plattformnutzern gesetzt werden. (3) Aufholen: Bei der WO-Strategie (Schwäche-Chancen-Kombination) wird die Schwäche, dass ein Betreiber einer Kunden-Plattform zunächst nicht im Besitz von großen Daten ist, mit der Chance der weiteren Etablierung als Datenökosystem kombiniert. In dieser Strategie besteht das Ziel in dem Ausbau einer engen und strategischen Kooperation mit bereits bestehenden, externen Plattformen für Datenlieferanten. Dadurch kann der Abwesenheit von Daten entgegengewirkt und der Zugang zu Daten aus diversen Branchen ermöglicht werden. (4) Vermeiden: Als WT-Strategie (Schwächen-Gefahren-Kombination) wird empfohlen, zunächst mit digitalen Pionieren, die über Know-How und Daten verfügen aus den verschiedenen Branchen zu kooperieren. Dadurch entstehen erste Leuchtturmprojekte, die Strahlkraft auf andere Unternehmen ausüben. Durch diese Strategie wird dem Ri-

Interne Sicht

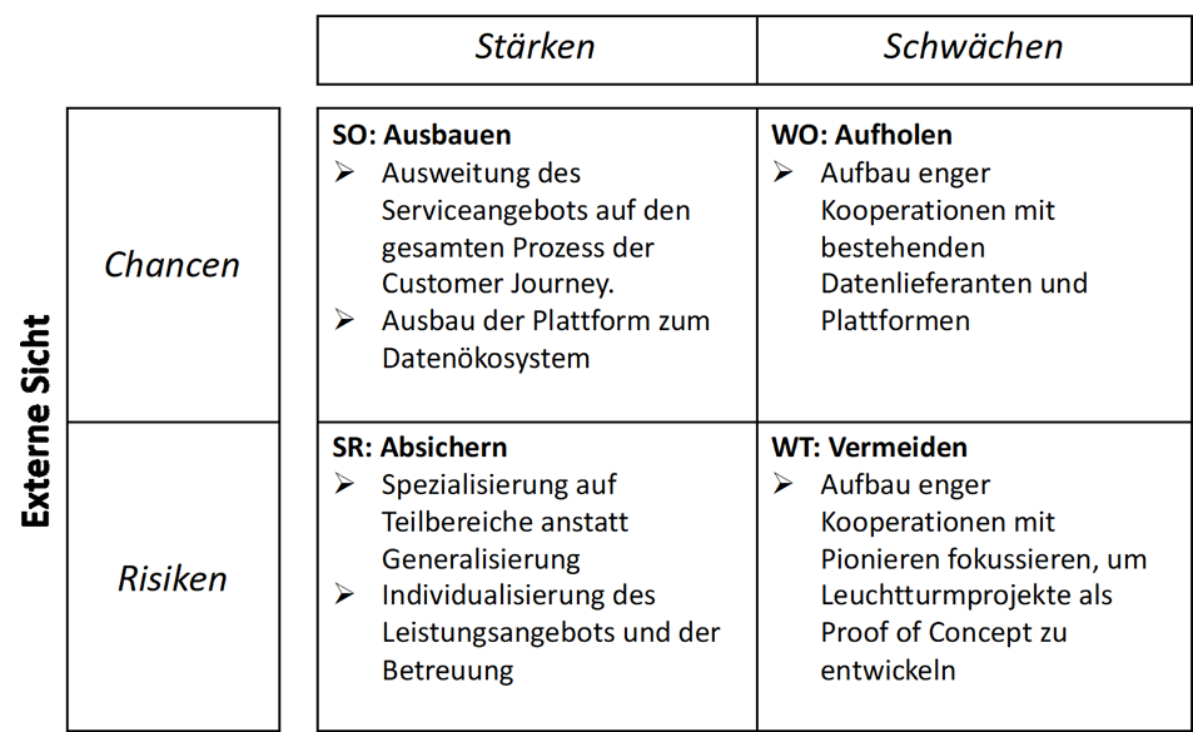

Abb. 4 Darstellung möglicher Strategien für das Geschäftsmodell 
siko der teilweise zu wenig digitalisierten Unternehmen sowie der Schwäche der unzureichenden Datenverfügbarkeit entgegengewirkt. In Abb. 4 sind die Strategien in einer Vier-Felder-Matrix zusammengefasst.

\section{Zusammenfassung und Ausblick}

In diesem Beitrag wurde zunächst exemplarisch demonstriert, wie eine KI-Applikation für ein Kundenbindungsszenario entwickelt werden kann. Durch die systematische Analyse des Datensatzes einer mittelständischen Kfz-Werkstatt und der Entwicklung eines KI-Modells lässt sich die durchschnittliche Fahrleistung von Kundenfahrzeugen prognostizieren, welche als Indikator für eine Kundenansprache verwendet werden kann und somit einen Beitrag zur Optimierung von Service- und Vertriebsprozessen leistet. Die Umsetzung der Fallstudie dient zum einen als Inspiration für Praktiker und zeigt Ansatzpunkte für den Einsatz von KI in Kundenbindungsszenarien auf. Anschließend erfolgte die induktive Ableitung eines plattformbasierten Geschäftsmodells für die zentrale Bereitstellung von KI-basierten Kundenbindungsservices. Die Wertschöpfung und Werteangebote des entwickelten Geschäftsmodells werden im Kern durch die intelligente Analyse von Daten erzeugt, wobei die Daten von Datenlieferanten bereitgestellt, die Analysen durch Datenbefähiger entwickelt und durch Datenkonsumenten nachgefragt werden. Stärken des Geschäftsmodells bestehen vor allem im hohen Skalierbarkeitspotential sowie den geringen Investitions- und Betriebskosten, wohingegen eine Schwäche z.B. im initial fehlenden Zugang zu Daten liegt. Damit das konzipierte Geschäftsmodell erfolgreich umgesetzt werden kann, ist der Zugang zu Datenquellen diverser Datenlieferanten unabdingbar. Zentraler Erfolgsfaktor ist daher die Weiterentwicklung der Plattform zu einem Datenökosystem durch die Gewinnung weiterer Akteure als Plattformnutzer. Datenökosysteme bergen in diesem Zusammenhang das Potenzial, den Austausch von Daten zu fördern und Angebot und Nachfrage zusammenzubringen (Oliveira und Lóscio 2018). Datenlieferanten, die bereit sind ihre Daten mit Serviceanbietern bzw. Datenbefähigern zu teilen, könnten die Serviceleistung beispielsweise zu einem reduzierten Preis beziehen oder an den Umsätzen des Serviceanbieters beteiligt werden. Auf diese Weise entsteht eine datenbasierte Wertschöpfungskette und das vorgestellte Geschäftsmodell schafft Mehrwerte für sämtliche Akteure des Datenökosystems (Ha et al. 2014).

Funding Open Access funding enabled and organized by Projekt DEAL.

Open Access Dieser Artikel wird unter der Creative Commons Namensnennung 4.0 International Lizenz veröffentlicht, welche die Nutzung, Vervielfältigung, Bearbeitung, Verbreitung und Wiedergabe in jeglichem Medium und Format erlaubt, sofern Sie den/die ursprünglichen Autor(en) und die Quelle ordnungsgemäß nennen, einen Link zur Creative Commons Lizenz beifügen und angeben, ob Änderungen vorgenommen wurden.

Die in diesem Artikel enthaltenen Bilder und sonstiges Drittmaterial unterliegen ebenfalls der genannten Creative Commons Lizenz, sofern sich aus der Abbildungslegende nichts anderes ergibt. Sofern das betreffende Material nicht unter der genannten Creative Commons Lizenz steht und die betreffende Handlung nicht nach gesetzlichen Vorschriften erlaubt ist, ist für die oben aufgeführten Weiterverwendungen des Materials die Einwilligung des jeweiligen Rechteinhabers einzuholen. 
Weitere Details zur Lizenz entnehmen Sie bitte der Lizenzinformation auf http://creativecommons.org/ licenses/by/4.0/deed.de.

\section{Literatur}

Alfs W, Deckers R (2020) Aftermarket 4.0 - Mit neuen Geschäftsmodellen und Services zum Erfolg. https://www.ifhkoeln.de/produkt/aftermarket-4-0/. Zugegriffen: 3. März 2021

Coleman S, Göb R, Manco G et al (2016) How can SMEs benefit from big data? Challenges and a path forward. Qual Reliab Eng Int 32(6):2151-2164. https://doi.org/10.1002/qre.2008

Gassmann O, Enkel E (2006) Open Innovation: Externe Hebeleffekte in der Innovation erzielen. Z Führung Org 75(3):132-138

Gomez-Uribe CA, Hunt N (2015) The Netflix recommender system: algorithms, business value and innovation. ACM Trans Manage Inf Syst. https://doi.org/10.1145/2843948

Ha S, Lee S-Y, Lee K (2014) Standardization requirements analysis on big data in public sector based on potential business models. Int J Softw Eng Appl 8(11):165-172

Könsgen R, Schaarschmidt M (2016) Key Performance Indicators für Software as a Service. HMD 53:662-673. https://doi.org/10.1365/s40702-016-0255-4

Lange J, Stahl F, Vossen G (2018) Datenmarktplätze in verschiedenen Forschungsdisziplinen: Eine Übersicht. Informatik Spektrum 41:170-180. https://doi.org/10.1007/s00287-017-1044-3

Lundberg S, Lee SI (2017) A unified approach to interpreting model predictions. arXiv preprint arXiv: 1705.07874

Oliveira MIS, Lóscio BF (2018) What is a data ecosystem? Proceedings of the 19th Annual Intern. Conference on Digital Government Research: Governance in the Data Age, S 1-9

Osterwalder A, Pigneur Y (2010) Business model generation: a handbook for visionaries, game changers, and challengers. John Wiley \& Sons

Overgoor G, Chica M, Rand W, Weishampel A (2019) Letting the computers take over: using AI to solve marketing problems. Calif Manage Rev 61(4):156-185. https://doi.org/10.1177/0008125619859318

Pérez J, Iturbide E, Olivares V et al (2015) A data preparation methodology in data mining applied to mortality population databases. In: Rocha A, Correia A, Costanzo S, Reis L (Hrsg) New contributions in information systems and technologies. Advances in intelligent systems and computing, Bd. 353. Springer, Cham, S 1173-1182

Rimbeck M, Reil H, Stumpf-Wollersheim J, Leyer M (2020) IoT-Geschäftsmodelle für Dienstleistungen in KMU. In: Automatisierung und Personalisierung von Dienstleistungen. Springer Gabler, Wiesbaden, S 313-335

Shearer C (2000) The CRISP-DM model: the new blueprint for data mining. J Data Warehous 5:13-22

Wellers D, Elliott T, Noga M (2017) ways machine learning is improving companies' work processes. Harvard Business Review. https://hbr.org/2017/05/8-ways-machine-learning-is-improving-companieswork-processes

Wiener M, Saunders C, Marabelli M (2020) Big-data business models: a critical literature review and multiperspective research framework. J Inf Technol 35(1):66-91. https://doi.org/10.1177/ 0268396219896811

Wilcoxon F, Katti SK, Wilcox RA (1970) Critical values and probability levels for the Wilcoxon rank sum test and the Wilcoxon signed rank test. American Cyanamid Company, Pearl River

Xia Y, Liu C, Li Y, Liu N (2017) A boosted decision tree approach using Bayesian hyper-parameter optimization for credit scoring. Expert Syst Appl 78:225-241 